\title{
The Quantified Level of Circulating Prostate Stem Cell Antigen mRNA relative to GAPDH Level Is a Clinically Significant Indictor for Predicting Biochemical Recurrence in Prostate Cancer Patients after Radical Prostatectomy
}

\author{
Sung Han Kim, ${ }^{1}$ Weon Seo Park, ${ }^{2}$ Sang Jin Lee, ${ }^{3}$ Moon Kyung Choi, ${ }^{4}$ \\ Seung Min Yeon, ${ }^{5}$ Jeong Nam Joo, ${ }^{5}$ Ara Ko, ${ }^{3}$ Eun Sik Lee, ${ }^{6}$ Jae Young Joung, ${ }^{1}$ \\ Ho Kyung Seo, ${ }^{1}$ Jinsoo Chung, ${ }^{1}$ and Kang Hyun Lee ${ }^{1}$ \\ ${ }^{1}$ Department of Urology, Center for Prostate Cancer, Research Institute and Hospital of National Cancer Center, \\ Goyang 410-769, Republic of Korea \\ ${ }^{2}$ Department of Pathology, Center for Prostate Cancer, Research Institute and Hospital of National Cancer Center, \\ Goyang 410-769, Republic of Korea \\ ${ }^{3}$ Genitourinary Cancer Branch, Center for Prostate Cancer, Research Institute and Hospital of National Cancer Center, \\ Goyang 410-769, Republic of Korea \\ ${ }^{4}$ Division of Specific Organ Cancer, Center for Prostate Cancer, Research Institute and Hospital of National Cancer Center, \\ Goyang 410-769, Republic of Korea \\ ${ }^{5}$ Biometric Research Branch, National Cancer Center, Goyang 410-769, Republic of Korea \\ ${ }^{6}$ Department of Urology, Seoul National University Hospital of Medicine, Seoul 110-744, Republic of Korea
}

Correspondence should be addressed to Kang Hyun Lee; uroonco@ncc.re.kr

Received 10 February 2015; Revised 9 May 2015; Accepted 13 May 2015

Academic Editor: Franco M. Buonaguro

Copyright (c) 2015 Sung Han Kim et al. This is an open access article distributed under the Creative Commons Attribution License, which permits unrestricted use, distribution, and reproduction in any medium, provided the original work is properly cited.

\begin{abstract}
The study quantified the relative absolute PSCA level in relation to the glyceraldehyde 3-phosphate dehydrogenase (GAPDH) level in the peripheral blood of 478 hormone-naive prostate cancer (PC) patients who underwent radical prostatectomy from 2005 to 2012 and evaluated its prognostic significance as a risk factor for predicting biochemical recurrence (BCR), compared to known parameters. Nested real-time polymerase chain reaction (RT-PCR) and gel electrophoresis detected PSCA levels and measured the PSCA/GAPDH ratio. Clinicopathological data from the institutional database were examined to determine the adequate cut-off level to predict postoperative BCR. A total of 110 patients had a positive PSCA result (23.0\%) via RT-PCR (mean blood ratio $1.1 \pm$ $0.4)$. The BCR was significantly higher in the PSCA-positive detection group $(p=0.009)$. A multivariate model was created to show that a PSCA/GAPDH ratio between 1.0 and 1.5 (HR 12.722), clinical T2c stage (HR 0.104), preoperative PSA (HR 1.225), extraprostatic capsule extension (HR 0.006), lymph node dissection (HR 16.437), and positive resection margin (HR 27.453) were significant predictive factors for BCR $(p<0.05)$. The study showed successful quantification of PSCA with its significance for BCR-related risk factor; however, further studies are needed to confirm its clinical predictive value.
\end{abstract}

\section{Introduction}

Prostate cancer (PC) has become the most frequent malignancy in men and causes the second highest number of cancer-related deaths. Half of all patients have metastatic disease at the initial diagnosis, and nearly half of the remainder who present with an initially localized disease will develop subsequent metastasis despite appropriate treatments [1]. To date, there have been no completely accurate diagnostic tools for controlling advanced disease states or predicting the 
early progression of PC during follow-ups; therefore, many clinicians are searching for new tumor markers or other methods to improve their detection rate.

Recent research has emphasized circulating tumor cells (CTCs), detected in the blood or lymphatic fluid from the primary PC lesion, as a potentially predictive micrometastatic tumor cell. This type of cell may metastasize to other organ sites when adequate conditions for survival are met in the secondary site. The CTC count is thus considered important for predicting PC progression, determining likely treatment outcomes, and choosing early preventive measures.

In particular, prostate stem cell antigen (PSCA) has recently come under scrutiny as a potential CTC marker. The antigen is found at low expression levels in the normal state but increases when the prostate's condition becomes malignant and during progression from an early to an advanced PC state $[2,3]$. The reverse transcriptase polymerase chain reaction (RT-PCR) has successfully quantified PSCA [4], prompting many other researchers to evaluate the benefits of PSCA as a diagnostic tool and explore its use in the treatment and prevention of PC in clinical and animal models [5-7].

Although this basic nested RT-PCR method for quantification of PSCA has not proven to be particularly precise upon repeated studies [8], a group from China recently used the RT-PCR method to show that circulating levels of PSCA correlated with androgen-independent progression in advanced PC [9]. Joung et al., from our institution, also showed the objective utility of using nested RT-PCR to quantify peripheral blood levels of PSCA mRNA to find several important genotypes and to predict the likelihood of biochemical recurrence (BCR) $[10,11]$.

Therefore, this study aimed to quantify absolute PSCA level in the peripheral blood of PC patients who underwent radical prostatectomy and relate it to a GADPH reference level (PSCA/GAPDH ratio) using RT-PCR. The study further aimed to determine PSCA's reliability as a tumor biomarker in comparison with other known clinicopathological prognostic parameters of BCR.

\section{Materials and Methods}

This study was approved by the Institutional Review Board (IRB number NCCNCS 05-049) with written consent from the participants. It was conducted according to the principles expressed in the Declaration of Helsinki.

2.1. Patient Selection and Blood Samples. From February 2005 to December 2012, 478 PC patients who underwent radical prostatectomy and standard pelvic lymph node dissection at the Center for Prostate Cancer, National Cancer Center, were prospectively selected for screening and possible inclusion into the study with a mean follow-up time of $42.1 \pm 25.30$ months. All cases were pathologically confirmed as adenocarcinomas, based on current World Health Organization criteria and Gleason grade by a single experienced uropathologist (Professor WSP, MD, PhD). Other clinicopathologic data were prospectively recorded in the Prostate Cancer Center database. No postoperative adjuvant hormonal or radiotherapies were performed until biochemical recurrence (BCR) developed, defined as a postoperative serum PSA elevation of $>0.2 \mathrm{ng} / \mathrm{mL}$ assessed on two occasions, following a prior $\mathrm{dec}-$ rease to nondetectable levels. The first PSA value of $0.2 \mathrm{ng} / \mathrm{mL}$ or greater was used to define the time of recurrence.

Researchers collected preoperative blood samples and performed RT-PCR to detect PSCA. A total of 135 (28.2\%) cases tested positive for PSCA. Exclusion criteria included refusal to participate in the study; missing preoperative clinicopathological data; inadequate volume of PSCA cDNA for RT-PCR; previous history of hormone therapy or chemotherapy or radiation therapy; history of invasive prostate treatment; postoperative loss to follow-up; or short follow-up less than 1 year. After excluding 25 patients based on these criteria, $110(23.0 \%)$ patients with PSCA-positive preoperative blood samples were ultimately enrolled.

\subsection{Prospectively Collected $m R N A$ and cDNA Extraction} and Reverse Transcription. Blood samples of $20 \mathrm{~mL}$ were obtained from patients just before the RP operation. Samples were delivered to the laboratory within an hour and nucleated cell fractions were isolated from $5 \mathrm{~mL}$ whole blood samples using Percoll Gradient Centrifugation (Amersham Biosciences, Uppsala, Sweden). Then, total RNA, extracted from nucleated cells, was converted to cDNA by reverse transcriptase and analyzed by PCR and nested PCR, as described in previous reports $[10,11]$. After RT-PCR assay sensitivity in cultured LNCaP cells was completed, assay products were evaluated to determine PSCA positivity. A 2\% agarose gel was used for electrophoresis, and an ultraviolet transilluminator (UV-T) was used for visualization. All 110 PSCA-positive samples were collected and stored at $-70^{\circ} \mathrm{C}$.

2.3. PSCA Quantification. The mRNA obtained from 110 PSCA-positive samples was converted to cDNA, and PCR/ nested PCR reactions from the prepared cDNA were utilized to calculate the amount of PSCA mRNA. The PSCA transcript quantities were normalized with an internal glyceraldehyde 3-phosphate dehydrogenase (GAPDH) mRNA control.

The PCR-reaction mixture for quantified analysis included $5 \mathrm{~L}$ of $\mathrm{cDNA}, 10 \mu \mathrm{L}$ of buffer (Go Taq), $0.25 \mu \mathrm{L}$ of DNA Polymerase (Promega Go Taq, Promega, WI), $4 \mu \mathrm{L}$ of dNTP $2.5 \mathrm{mM}$ mixture (TaKaRa, Japan), $\leq 50 \mu \mathrm{L}$ of distilled water, and $1 \mu \mathrm{L}$ of each primer.

All primers used in this study were synthesized by Bioneer (Daejeon, Korea). The primers specific for human PSCA were $5^{\prime}$-CCC TGC AGC CAG GCA CT- $3^{\prime}$ and $5^{\prime}$-AGG CCA ACT GCG CG AT- $5^{\prime}$. Primers for human GAPDH were $5^{\prime}$ TGG TCA CCA GGG CTG CTT TTA- $3^{\prime}$ and $5^{\prime}$-TCC TGG AAG ATG GTG ATG GGA TTT-3'. For nested PCR for PSCA detection, primers were $5^{\prime}$-CAC TGC CCT GCT GTG CTA CT- $3^{\prime}$ and $5^{\prime}$-CGC GGT CCA GCA CTG CTC CC- $3^{\prime}$. For the internal control to assess RNA integrity, GAPDH was amplified. PCR reactions were performed in a total volume of $50 \mu \mathrm{L}$ containing $1 \mu \mathrm{L}$ of RT product, $5 \mu \mathrm{L}$ of $10 \mathrm{x}$ PCRreaction buffer $(200 \mathrm{mmol} / \mathrm{L}$ Tris $\mathrm{HCl}, \mathrm{pH} 8.4,500 \mathrm{mmol} / \mathrm{L}$ $\mathrm{KCl}), 0.1 \mu \mathrm{mol} / \mathrm{L}$ sense and $0.1 \mu \mathrm{mol} / \mathrm{L}$ antisense primer, $0.2 \mathrm{mmol} / \mathrm{L}$ of each $\mathrm{dNTP}$, and $1 \mathrm{U}$ Taq-DNA polymerase. 
Amplification of $5 \mu \mathrm{L}$ cDNA was performed using a Tube Controlled Thermal Cycler (MJ Research, Waltham, MN, USA) with the following reaction profile: $2 \mathrm{~min}$ of initial denaturation at $95^{\circ} \mathrm{C}$ followed by single cycle of denaturation at $95^{\circ} \mathrm{C}$ for $30 \mathrm{~s} ; 30 \mathrm{~s}$ of annealing at $55^{\circ} \mathrm{C}$ followed by 35 cycles at $94^{\circ} \mathrm{C}$ for $30 \mathrm{~s}, 55^{\circ} \mathrm{C}$ for $1 \mathrm{~min}$, and $72^{\circ} \mathrm{C}$ for $1 \mathrm{~min}$; and a final extension at $72^{\circ} \mathrm{C}$ for $5 \mathrm{~min}$. For nested PCR, $5 \mu \mathrm{L}$ of PCR product was amplified with the same reagents and with nested primers for an additional 35 cycles. LNCaP cells were cultured, and RT-PCR assay sensitivity was determined, as described in a previous report $[10,11]$. The sensitivity of LNCaP cells and other markers were all analyzed to confirm the amount of PSCA transcripts using GADPH as a reference.

Quantification was performed on $1 \%$ agarose gel treated with $0.5 \mu \mathrm{g} / \mathrm{mL}$ ethidium bromide stain. Then, the UV-T was used to visualize the fluorescent expression of PSCA and GAPDH, and the relative amount of PSCA transcripts was determined.

2.4. Statistical Analysis. The chi-squared and Fisher's exact tests were used to evaluate the correlation of PSCA titer and PSCA/GAPDH ratio with clinicopathological factors. Student's $t$-test was used to compare differences in BCR. In this study, disease progression was defined as BCR after prostatectomy. Multivariate survival analysis was evaluated using Cox's proportional hazard models to identify independent prognostic factors of BCR. Two-sided $p$ values $<0.05$ using STATA (release 9.2, STATA Inc., TX, USA) were considered statistically significant.

\section{Results}

The clinicopathologic characteristics and demographics of the 110 positive PSCA patients are described in Table 1. Using nested RT-PCR, a mean PSCA titer of $9531.2 \pm 4272.5$ was detected, and a relative mean PSCA/GADPH ratio of $1.1 \pm$ 0.4 was detected via gel electrophoresis and UV-T (Table 1 , Figure 1).

The correlation analysis of PSCA titers and known clinicopathologic prognostic parameters showed that clinical (odds ratio [OR] 15.049) and pathologic $\mathrm{T}$ stage presence (OR 8.431), a positive resection margin (OR 9.545), and apical tumor involvement (OR 13.291) were significantly correlated with PSCA titer, and BCR (OR 8.091) and perineural invasion (OR 8.233) were significantly correlated with the PSCA/ GAPDH ratio ( $p<0.05$, Table 2$)$. The BCR-free survival curve showed that PSCA-positive group had higher incidence of BCR than the negative group ( $p=0.009$, Figure 2 ).

To determine the PSCA/GAPDH ratio as a predictive prognostic factor for $\mathrm{BCR}$, univariate and multivariate analyses were performed. The univariate analysis showed that the preoperative PSA, PSCA absolute titer, PSCA/GAPDH ratio, pathologic Gleason score sum, clinical T stage, positive resection margin, lymphovascular invasion, perineural invasion, extraprostatic capsule extension, seminal vesicle invasion, lymph nodal dissection, and pathologic $\mathrm{T}$ stage were significantly different ( $p<0.05$, data not shown). The multivariate Cox regression model showed that a PSCA/GAPDH ratio of 1.0-1.5 (HR 12.722, 95\% CI 1.737-843.933), a clinical T2c stage
TABle 1: Patients' clinicopathologic characteristics $(N=110)$.

\begin{tabular}{|c|c|}
\hline Parameters & $N(\%)$ or mean $\pm \mathrm{SD}$ \\
\hline Preoperative PSA (ng/mL) & $11.7 \pm 10.3$ \\
\hline Prostate volume $(\mathrm{mL})$ & $35.5 \pm 16.1$ \\
\hline PSCA absolute titer & $9531.2 \pm 4272.5$ \\
\hline$>15,000$ & $9(8.2)$ \\
\hline $10,1001-1,5000$ & $38(34.5)$ \\
\hline $5001-10,000$ & $51(46.4)$ \\
\hline$<5000$ & $12(10.9)$ \\
\hline PSCA/GAPDH ratio & $1.1 \pm 0.4$ \\
\hline$>1.5$ & $11(10.0)$ \\
\hline $1.1-1.5$ & $56(50.9)$ \\
\hline $0.5-1.0$ & $35(31.8)$ \\
\hline$>0.5$ & $8(7.3)$ \\
\hline Gleason score sum & $6.6 \pm 0.8$ \\
\hline Clinical stage $\mathrm{T} 1$ & $36(32.7)$ \\
\hline $\mathrm{T} 2$ & $56(50.9)$ \\
\hline $\mathrm{T} 3$ & $16(14.6)$ \\
\hline N1 & $2(1.8)$ \\
\hline Pathologic Gleason score sum & $7.0 \pm 0.9$ \\
\hline Pathologic prostate volume $(\mathrm{mL})$ & $33.8 \pm 15.2$ \\
\hline Pathologic stage $\leq \mathrm{T} 2 \mathrm{~b}$ & $63(57.3)$ \\
\hline$\geq \mathrm{T} 2 \mathrm{c}$ & $47(42.7)$ \\
\hline $\mathrm{N}(+)$ & $1(0.9)$ \\
\hline M1 & $4(3.6)$ \\
\hline Biochemical recurrence & $24(21.8)$ \\
\hline $\begin{array}{l}\text { Median time to biochemical recurrence } \\
\text { (months) }\end{array}$ & $7.0(3-46)$ \\
\hline Median follow-up duration (months) & $42.1 \pm 25.0$ \\
\hline
\end{tabular}

(HR 0.104, 95\% CI 0.018-0.621), preoperative PSA levels (HR 1.225, 95\% CI 1.068-1.406), extraprostatic capsule extension (HR 0.006, 95\% CI 0.001-0.271), lymph node dissection (HR 16.437, 95\% CI 1.024-263.859), and positive resection margin (HR 27.453, 95\% CI 3.5-215.318) remained significant risk factors for BCR $(p<0.05$, Table 3$)$.

\section{Discussion}

A blood-based examination of PSA has historically been the most popular diagnostic screening tool for predicting PC risk and outcome; however, it is difficult to accurately predict the progression of advanced and localized PC even after adequate treatment based solely on PSA results, because the hormone's values can fluctuate up to $20-30 \%$ based on biological and environmental factors [12]. Therefore, many clinicians and researchers have suggested alternative prognostic measurements and markers such as derivatives of PSA kinetics, other imaging modalities, or novel tumor biomarkers to supplement or fully replace PSA and better predict PC prognosis and progression [13].

Recently, focus has shifted to micrometastatic CTC biomarkers in peripheral blood [13-15]. In particular, PSCA has 
TABLE 2: Correlation of prostate stem cell antigen titer with known clinicopathological prognostic parameters $(B=110)$.

\begin{tabular}{|c|c|c|}
\hline Parameters & $\begin{array}{l}\text { Correlation with PSCA absolute titer } \\
\text { ( } p \text { value })\end{array}$ & $\begin{array}{c}\text { Correlation with PSCA/GAPDH } \\
\text { ( } p \text { value })\end{array}$ \\
\hline Age (years) & 0.601 & 0.685 \\
\hline Preoperative PSA (ng/mL) & 0.457 & 0.639 \\
\hline Preoperative-free PSA (ng/DL) & 0.357 & 0.458 \\
\hline Prostate size $(\mathrm{mL})$ & 0.458 & 0.385 \\
\hline Gleason score sum at biopsy & 0.508 & 0.741 \\
\hline Clinical T stage $(\mathrm{T} 1, \mathrm{~T} 2, \geq \mathrm{T} 3)$ & 0.010 (OR 15.049) & 0.485 \\
\hline Positive resection margin & 0.018 (OR 9.545) & 0.125 \\
\hline Pathologic Gleason score sum & 0.630 & 0.571 \\
\hline Pathologic T stage $(\leq \mathrm{T} 2 \mathrm{~b}$ or $\geq \mathrm{T} 2 \mathrm{c})$ & $0.033($ OR 8.431$)$ & 0.187 \\
\hline Pathologic $\mathrm{N}$ or $\mathrm{M}$ stage & 0.234 & 0.259 \\
\hline Apex involvement & 0.003 (OR 13.291) & 0.643 \\
\hline Lymphovascular invasion & 0.643 & $0.053($ OR 7.934$)$ \\
\hline Perineural invasion & 0.059 (OR 7.419) & 0.039 (OR 8.233) \\
\hline Extracapsular extension & 0.051 (OR 7.658) & 0.108 \\
\hline Seminal vesicle invasion & 0.810 & 0.950 \\
\hline Biochemical recurrence & 0.681 & 0.032 (OR 8.091) \\
\hline
\end{tabular}

OR: odds ratio; HGPIN: high grade prostatic intraepithelial neoplasm; PSA: prostatic specific antigen.
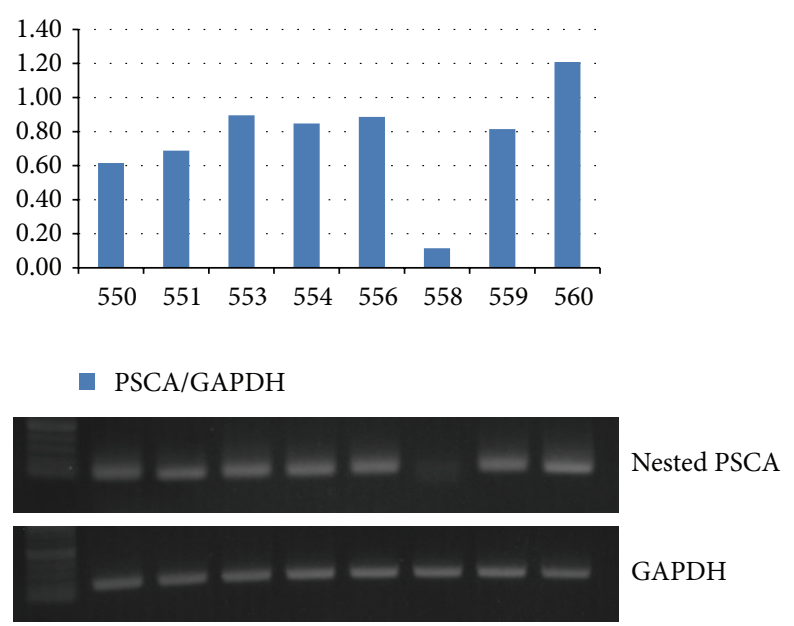

M $\quad \begin{array}{lllllllll}550 & 551 & 553 & 554 & 556 & 558 & 559 & 560 & \text { RP sample number }\end{array}$

FIGURE 1: Detection of PSCA and GAPDH positivity using gel electrophoresis with ultraviolet transilluminator.

received recent scrutiny as a biomarker because it can accurately define the correlations between PC prognosis, diagnosis, and preventive measures [16, 17]. Highly overexpressed in human PC but with only limited expression in normal tissues, PSCA is a cell-surface antigen that belongs to the Ly-6/Thy1 family of glycosylphosphatidylinositol-anchored proteins. The function of PSCA in normal and tumor contexts is not perfectly known; however, the Thy-1 family is known to play a role in $\mathrm{T}$ cell activation, proliferation, stem cell survival, and cytokine and growth factor responses. Furthermore, PSCA

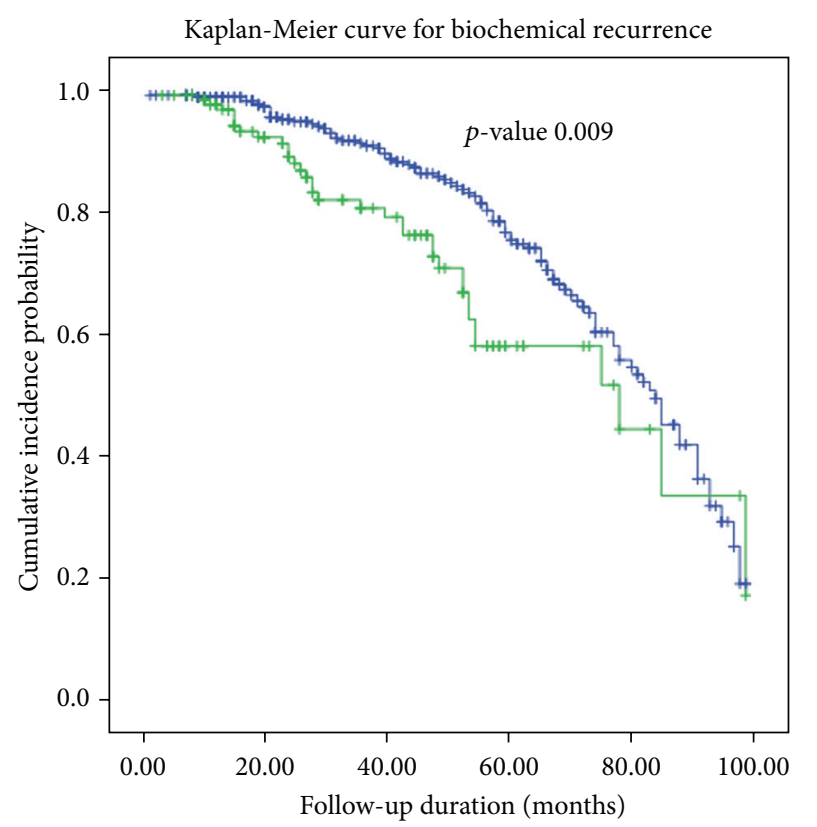

PSCA_CTC

$\neg$ PSCA-negative group $\quad+$ Negative group-censored for BCR

$\neg$ PSCA-positive group $\quad+$ Positive group-censored for BCR

FIGURE 2: Kaplan-Meier curve for biochemical recurrence-free survival between PSCA-negative and PSCA-positive groups.

has already been proven to play a specific role in and have a strong relationship with PC development, because the Ly6 family is associated with carcinogenesis, cellular activation, and cell adhesion of tumor cells $[2,18,19]$. 
TABLE 3: Multivariate analysis of BCR-related risk factors using stratified PSCA/GAPDH ratio into three subgroups.

\begin{tabular}{|c|c|c|c|c|}
\hline & \multirow{2}{*}{$p$ value } & \multirow{2}{*}{ Hazard ratio } & \multicolumn{2}{|c|}{$95.0 \%$ confidence interval } \\
\hline & & & Lower limit & Upper limit \\
\hline Lymphovascular invasion & 0.072 & 0.059 & 0.003 & 1.293 \\
\hline Perineural invasion & 0.721 & 0.612 & 0.042 & 9.024 \\
\hline Extraprostatic capsule extension & 0.008 & 0.006 & 0.001 & 0.271 \\
\hline Seminal vesicle invasion & 0.589 & 1.464 & 0.367 & 5.838 \\
\hline Lymph node dissection & 0.048 & 16.437 & 1.024 & 263.859 \\
\hline Resection margin negative & 0.002 & 27.453 & 3.500 & 215.318 \\
\hline Neurovascular saving & 0.110 & 4.217 & 0.723 & 24.603 \\
\hline Clinical T1 & 0.006 & & & \\
\hline cT2a, T2b & 0.165 & 0.077 & 0.002 & 2.864 \\
\hline $\mathrm{cT} 2 \mathrm{c}$ & 0.013 & 0.104 & 0.018 & 0.621 \\
\hline$\geq \mathrm{cT} 3$ & 0.083 & 14.700 & 0.706 & 306.063 \\
\hline Pathologic Gleason score & 0.801 & 1.256 & 0.213 & 7.397 \\
\hline PSCA/GAPDH > 1.5 & 0.052 & & & \\
\hline $1.0-1.5$ & 0.016 & 12.722 & 1.598 & 101.286 \\
\hline$<1.0$ & 0.135 & 5.403 & 0.590 & 49.499 \\
\hline Preoperative PSA & 0.004 & 1.225 & 1.068 & 1.406 \\
\hline Pathologic $\leq \mathrm{T} 2 \mathrm{~b}$ & 0.494 & 0.403 & 0.030 & 5.468 \\
\hline
\end{tabular}

Our institution has been conducting prospective studies to find potential tumor biomarkers in the peripheral blood of Korean PC patients since 2005, and we have further reinforced the potential of PSCA as a potential PC tumor biomarker $[10,11]$. The marker has already been proven to have a significant correlation with prognostic parameters of BCR, and the genetic specificity of haplotype increased the risk of PC prevalence. As our previous studies qualitatively defined PSCA as a potential PC biomarker after radical prostatectomy, we conducted this study to definitively quantify the clinical usefulness of PSCA titer in blood samples by more accurately defining the PSCA/GAPDH ratio as a significant prognostic tumor biomarker for BCR.

In this study, $28.2 \%(N=135)$ of peripheral blood samples from 478 patients' blood samples were positive for PSCA, and $23.0 \%(N=110)$ of samples were ultimately include. The included patients had significantly lower rates of BCRfree survival compared to others in the Kaplan-Meier plot, assessed via the log rank test ( $p=0.009$, Figure 2 ). The quantified PSCA/GAPDH ratio was analyzed in both correlation and multivariate models to determine its potential predictive risk factor for BCR (Tables 2 and 3). The analyses showed that the PSCA/GAPDH ratio was related to BCR and its stratified value of 1.0-1.5 (HR 12.722) was found significant for predicting BCR after radical prostatectomy $(p<0.05)$ with other significant clinical T2c stage (HR 0.104), lymph node dissection (HR 16.437), positive resection margin (HR 27.453), extraprostatic capsule extension (HR 0.006), and preoperative PSA (HR 1.225), which have been already shown in previous studies $(p<0.05$, Table 3$)$ [20-22].

Background analyses were performed to find an appropriate discriminating cut-off value and its clinical value in predicting the BCR. An arbitrary cut-off value of 1.2 around a median value between 1.0 and 1.5 showed a statistical discriminating power of BCR-free survival curve in the KaplanMeier curve with log rank test $(p=0.034$, not shown in figure). However, this value was not finally proven to be valid for determining the statistical significance because external validation could not be performed and internal validation failed due to the limited number. Although this study has limited generalizability in defining a specific value of PSCA/GAPDH ratio due to the limited number of samples and lack of internal and external validations, this study is clinically significant because, for the first time, a study successfully suggested that quantified PSCA with a significant range value could be a potential prognostic factor for BCR and an alternative followup biomarker to PSA after radical prostatectomy.

Most previous PSCA studies have been based on Western patients or animal modeling studies, and only a few Asian studies have reported PSCA as a tumor biomarker strongly associated with clinicopathological parameters such as the Gleason score, pathologic stage, seminal vesicle invasion, extraprostatic capsule extension, and metastasis [4, 9, 23]. Their findings of PSCA correlation to disease invasiveness and progression were similar to our findings (Table 2). Unfortunately, however, none of those previous studies were prospectively designed, involved large numbers of samples from PC patients underwent radical prostatectomy, or involved long-term follow-up and they did not investigate PSCA's genetic, qualitative, and quantitative aspects, as was done in the present study and our previous studies $[10,11]$. Thus, no definitive conclusions can yet be made, although results remain encouraging. Additionally, the subanalytic correlation study of stratified PSA, Gleason score, and pathologic T stage showed that none of the significant stratified groups of PSA, Gleason score, and pathologic T stage were significantly 
related to the PSCA/GAPDH ratio $(p>0.05$, data not shown). The PSCA/GAPDH ratio would thereby be an independent biomarker for these three markers.

The limitations of this study included the retrospective nature of the quantitative analysis, the inaccuracy of nested RT-PCR using UV-T for quantification of PSCA positivity, and no internal and external validation of our arbitrary PSCA/GAPDH cut-off value. Further larger analyses with more sensitive and accurate modalities to detect the quantified PSCA in blood samples, such as recently introduced detection equipment using nanotechnologies, are required. Additionally, longer-term follow-up is necessary to fully evaluate the prognostic value of PSCA including overall survival and progression-free survival to hormone refractory state in PC.

\section{Conclusion}

The study showed that a patient's quantified PSCA ratio to GAPDH (PSCA/GAPDH ratio) was significantly correlated with BCR and if its value was between 1.0 and 1.5, it was a significant risk factor for BCR. This suggested the possibilities of using PSCA/GAPDH ratio as an alternative tool for prognosis prediction than what has been examined to date. Further studies with more accurate analytic methods should be considered to detect the titers of PSCA in the peripheral blood of PC patients as an alternative to current PSA tests.

\section{Abbreviations}

cDNA: Complementary DNA

dNTP: Deoxynucleotide triphosphate

LNCaP: Oncological cell line

mRNA: Messenger RNA.

\section{Conflict of Interests}

The authors declare that there is no conflict of interests regarding the publication of this paper.

\section{Acknowledgments}

Ms. Jung Eun Kim from the Prostate Cancer Department contributed to the database management. This work was supported by a grant from the National Cancer Center (NCC1210110 and NCC-1510170), Republic of Korea.

\section{References}

[1] R. Siegel, D. Naishadham, and A. Jemal, "Cancer statistics, 2013," CA: Cancer Journal for Clinicians, vol. 63, no. 1, pp. 11-30, 2013.

[2] R. E. Reiter, Z. Gu, T. Watabe et al., "Prostate stem cell antigen: a cell surface marker overexpressed in prostate cancer," Proceedings of the National Academy of Sciences of the United States of America, vol. 95, no. 4, pp. 1735-1740, 1998.

[3] A. C. Cunha, B. Weigle, A. Kiessling, M. Bachmann, and E. P. Rieber, "Tissue-specificity of prostate specific antigens: comparative analysis of transcript levels in prostate and non-prostatic tissues," Cancer Letters, vol. 236, no. 2, pp. 229-238, 2006.
[4] N. Hara, T. Kasahara, T. Kawasaki et al., "Reverse transcriptionpolymerase chain reaction detection of prostate-specific antigen, prostate-specific membrane antigen, and prostate stem cell antigen in one milliliter of peripheral blood: value for the staging of prostate cancer," Clinical Cancer Research, vol. 8, no. 6, pp. 1794-1799, 2002.

[5] M. L. Moore, M. A. Teitell, Y. Kim et al., "Deletion of PSCA increases metastasis of TRAMP-induced prostate tumors without altering primary tumor formation," The Prostate, vol. 68, no. 2, pp. 139-151, 2008.

[6] J. S. Lam, J. Yamashiro, I. P. Shintaku et al., "Prostate stem cell antigen is overexpressed in prostate cancer metastases," Clinical Cancer Research, vol. 11, no. 7, pp. 2591-2596, 2005.

[7] K.-R. Han, D. B. Seligson, X. Liu et al., "Prostate stem cell antigen expression is associated with gleason score, seminal vesicle invasion and capsular invasion in prostate cancer," The Journal of Urology, vol. 171, no. 3, pp. 1117-1121, 2004.

[8] D. Schamhart, J. Swinnen, K.-H. Kurth et al., "Numeric definition of the clinical performance of the nested reverse transcription-PCR for detection of hematogenous epithelial cells and correction for specific mRNA of non-target cell origin as evaluated for prostate cancer cells," Clinical Chemistry, vol. 49, no. 9, pp. 1458-1466, 2003.

[9] Z. Zhao, G. Zeng, W. Ma, L. Ou, and Y. Liang, "Peripheral blood reverse transcription PCR assay for prostate stem cell antigen correlates with androgen-independent progression in advanced prostate cancer," International Journal of Cancer, vol. 131, no. 4, pp. 902-910, 2012.

[10] J. Y. Joung, K. S. Cho, J. E. Kim et al., "Prostate stem cell antigen mRNA in peripheral blood as a potential predictor of biochemical recurrence in high-risk prostate cancer," Journal of Surgical Oncology, vol. 101, no. 2, pp. 145-148, 2010.

[11] J. Y. Joung, S. O. Yang, I. G. Jeong et al., "Reverse transcriptasepolymerase chain reaction and immunohistochemical studies for detection of prostate stem cell antigen expression in prostate cancer: potential value in molecular staging of prostate cancer," International Journal of Urology, vol. 14, no. 7, pp. 635-643, 2007.

[12] G. Sölétormos, A. Semjonow, P. E. C. Sibley et al., "Biological variation of total prostate-specific antigen: a survey of published estimates and consequences for clinical practice," Clinical Chemistry, vol. 51, no. 8, pp. 1342-1351, 2005.

[13] C. Stephan, H. Cammann, H.-A. Meyer, M. Lein, and K. Jung, "PSA and new biomarkers within multivariate models to improve early detection of prostate cancer," Cancer Letters, vol. 249, no. 1, pp. 18-29, 2007.

[14] S. F. Shariat, J. A. Karam, and C. G. Roehrborn, "Blood biomarkers for prostate cancer detection and prognosis," Future Oncology, vol. 3, no. 4, pp. 449-461, 2007.

[15] G. Sardana and E. P. Diamandis, "Biomarkers for the diagnosis of new and recurrent prostate cancer," Biomarkers in Medicine, vol. 6, no. 5, pp. 587-596, 2012.

[16] S. Ross, S. D. Spencer, I. Holcomb et al., "Prostate stem cell antigen as therapy target: tissue expression and in vivo efficacy of an immunoconjugate," Cancer Research, vol. 62, no. 9, pp. 2546-2553, 2002.

[17] A. B. Raff, A. Gray, and W. M. Kast, "Prostate stem cell antigen: a prospective therapeutic and diagnostic target," Cancer Letters, vol. 277 , no. 2, pp. 126-132, 2009.

[18] I. P. Witz, "Differential expression of genes by tumor cells of a low or a high malignancy phenotype: the case of murine and human Ly-6 proteins," Journal of Cellular Biochemistry. Supplement, vol. 77, no. 34, pp. 61-66, 2000. 
[19] T. A. Rege and J. S. Hagood, "Thy-1, a versatile modulator of signaling affecting cellular adhesion, proliferation, survival, and cytokine/growth factor responses," Biochimica et Biophysica Acta, vol. 1763, no. 10, pp. 991-999, 2006.

[20] T. Koie, K. Mitsuzuka, T. Yoneyama et al., "Prostate-specific antigen density predicts extracapsular extension and increased risk of biochemical recurrence in patients with high-risk prostate cancer who underwent radical prostatectomy," International Journal of Clinical Oncology, vol. 20, no. 1, pp. 176-181, 2015.

[21] O. Yossepowitch, A. Briganti, J. A. Eastham et al., "Positive surgical margins after radical prostatectomy: a systematic review and contemporary update," European Urology, vol. 65, no. 2, pp. 303-313, 2014.

[22] S. Punnen, M. R. Cooperberg, A. V. D’Amico et al., "Management of biochemical recurrence after primary treatment of prostate cancer: a systematic review of the literature," European Urology, vol. 64, no. 6, pp. 905-915, 2013.

[23] Z. Zhigang and S. Wenlu, "The association of prostate stem cell antigen (PSCA) mRNA expression and subsequent prostate cancer risk in men with benign prostatic hyperplasia following transurethral resection of the prostate," The Prostate, vol. 68, no. 2, pp. 190-199, 2008. 


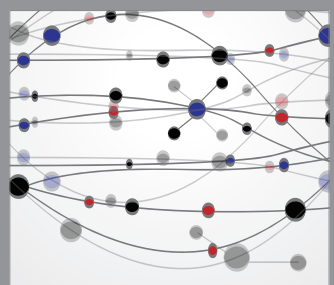

The Scientific World Journal
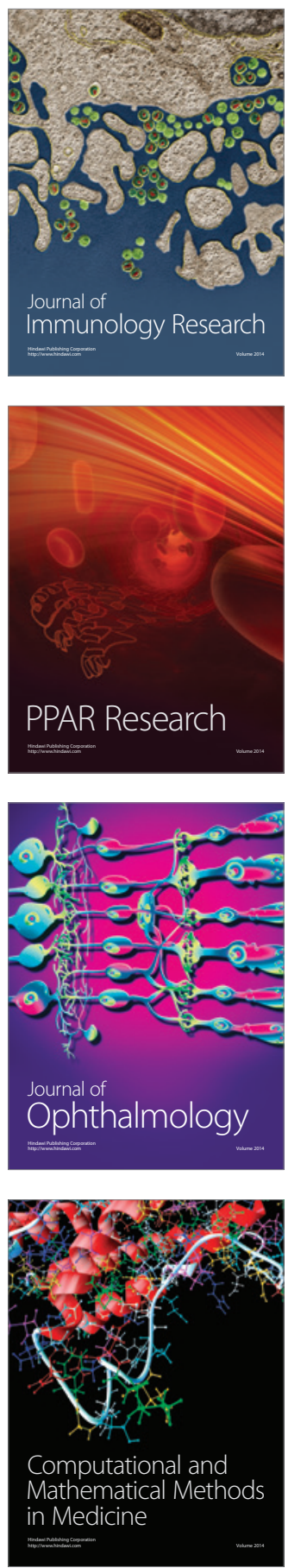

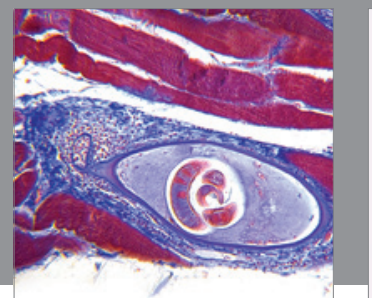

Gastroenterology

Research and Practice
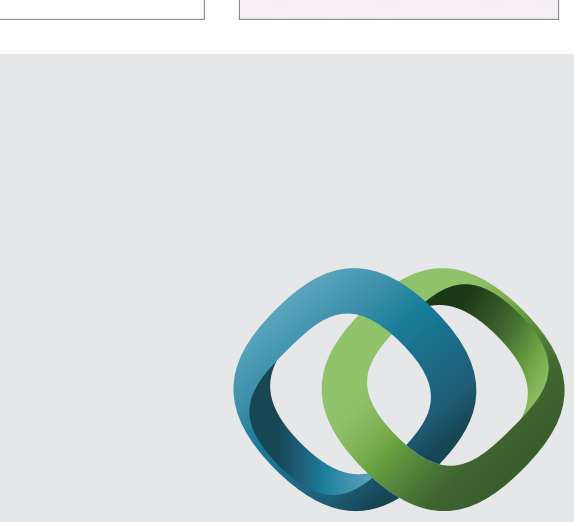

\section{Hindawi}

Submit your manuscripts at

http://www.hindawi.com
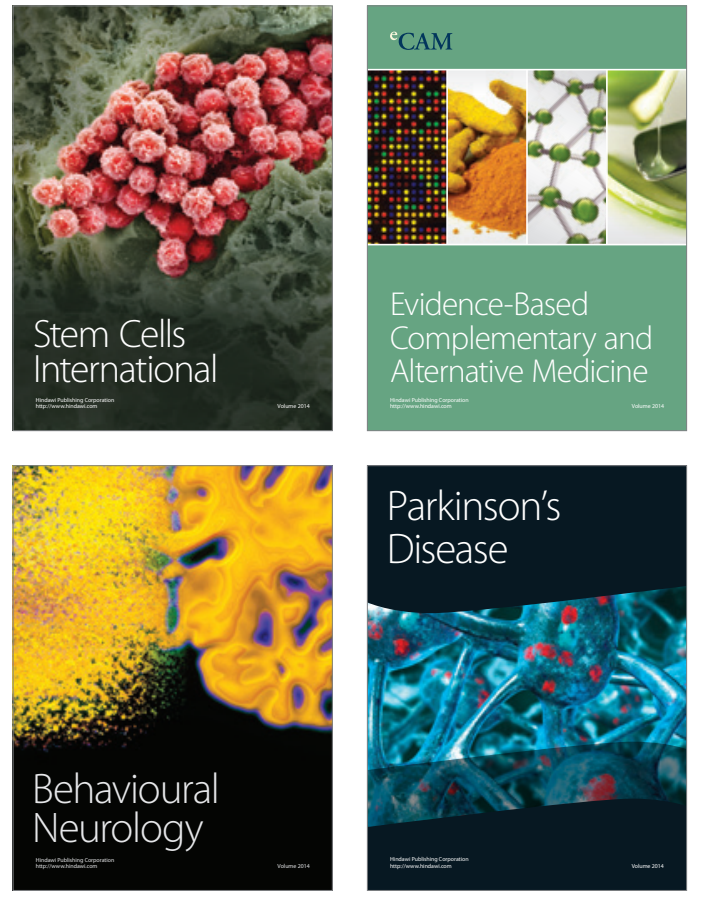
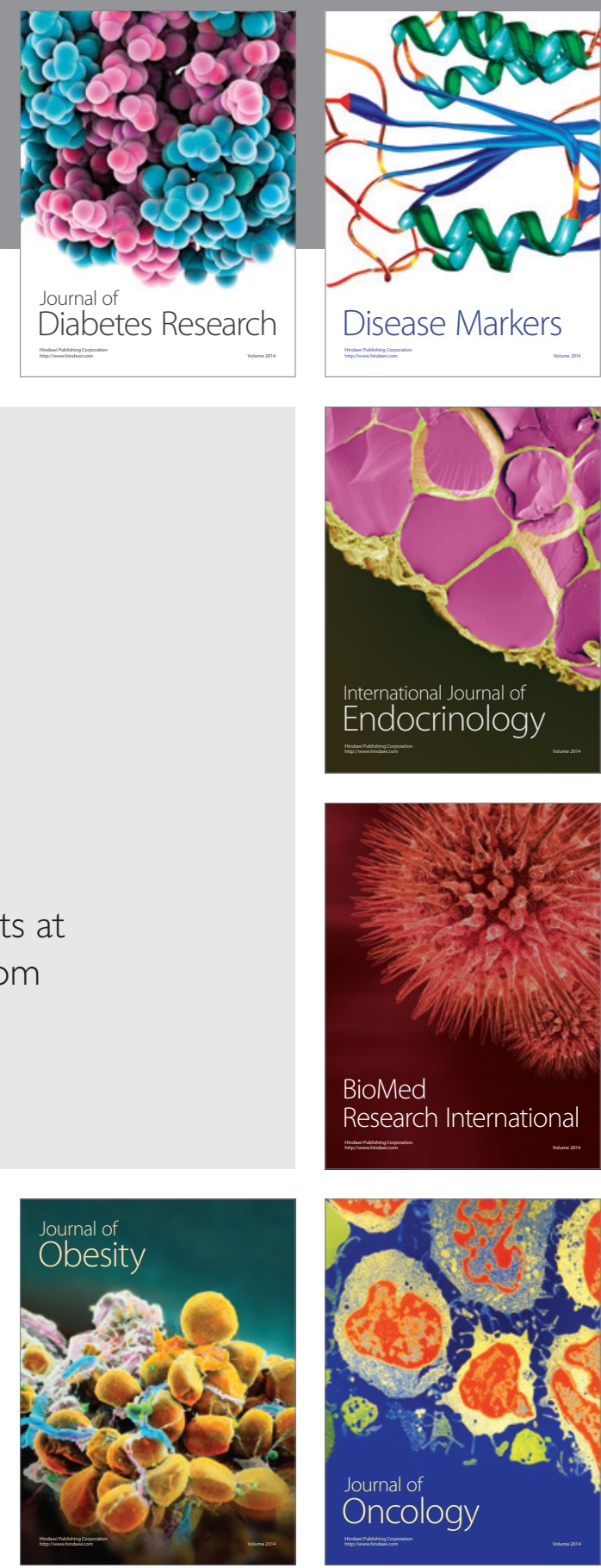

Disease Markers
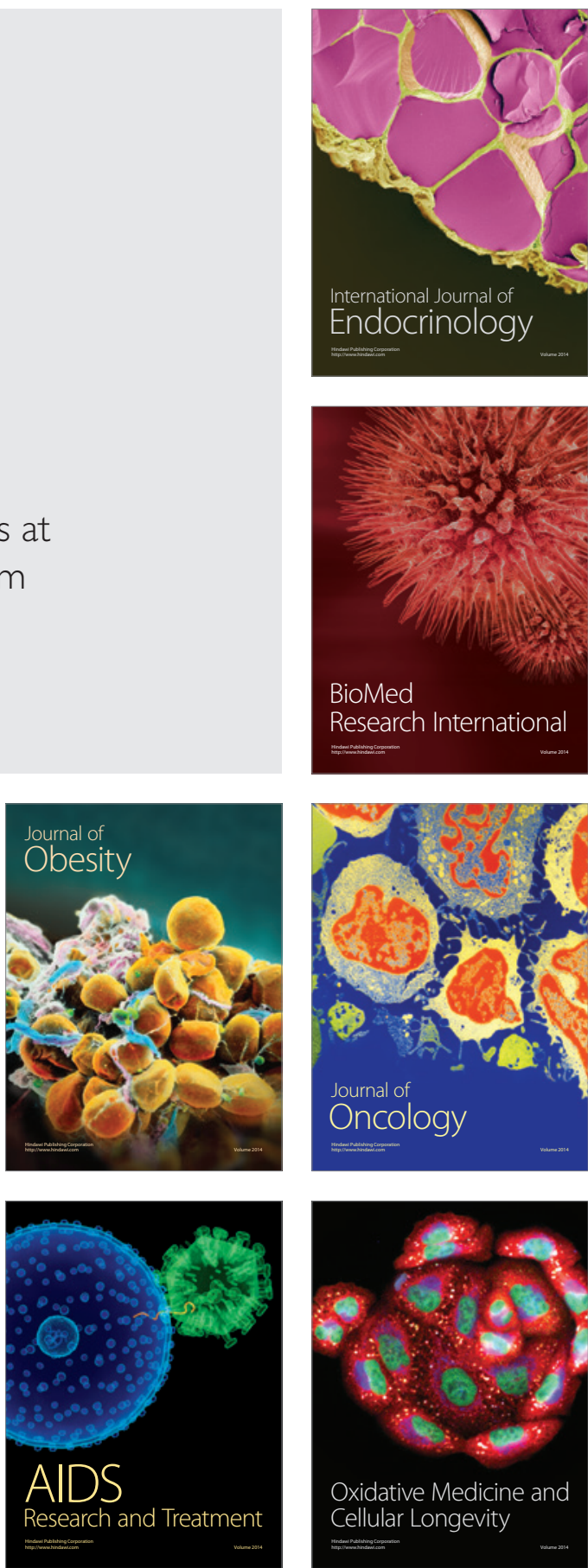